between, but the situation is sufficiently serious for key stakeholders to have begun to take notice and to introduce measures promoting robust design and transparent reporting. The NIH now has dedicated sections in grant proposals for applicants to describe how they will ensure their findings are robust, and Nature has introduced reporting checklists for submitted papers. There is growing interest in 'open science' - championed by the Center for Open Science in Charlottesville, Virginia - whereby elements of the research process (such as protocols, materials or data) are made publicly available. One positive outcome of the growth in metascience is that it has highlighted how every field typically does something very well, from preregistration to data sharing.

It is ironic that scientists in the pharmaceutical industry - often the target of opprobrium and worries about conflicts of interest - were among the first to raise concerns about the functionality of biomedical science. But it isn't surprising. They have incentives to be right - to make a correct 'go' decision on a compound that proves to be a successful treatment. Academic scientists, by contrast, are incentivized to publish first, to get grants and so on, but only rarely to get the right answer. In the words of Veronique Kiermer, executive editor at the Public Library of Science in San Francisco, California, "It actually pays to be sloppy and just cut corners and get there first". So what is good for scientists' careers may not be good for science. Simulations support this, suggesting that labs that do sloppy science will 'outperform' more-rigorous ones.

Harris makes a strong case that the biomedical research culture is seriously in need of repair. His focus is on preclinical research (and is rather US-centric), but he ends on a more optimistic note. The culture in various branches of biomedical science is changing, and incorporating lessons from other branches - preregistration of protocols, reporting checklists, and open data and materials. There is also cross-pollination of ideas between academia and industry. And funders and journals have begun initiatives to improve the quality of research.

Looked at in this way, biomedical research is not in crisis, but is embracing an opportunity to improve how it works, using scientific tools to understand the scientific process. Change takes time; Rigor Mortis shows that reproducibility issues are now mainstream, and that can only be good for science.

Marcus Munafò is professor of biological psychology at the University of Bristol, UK. e-mail:marcus.munafo@bristol.ac.uk

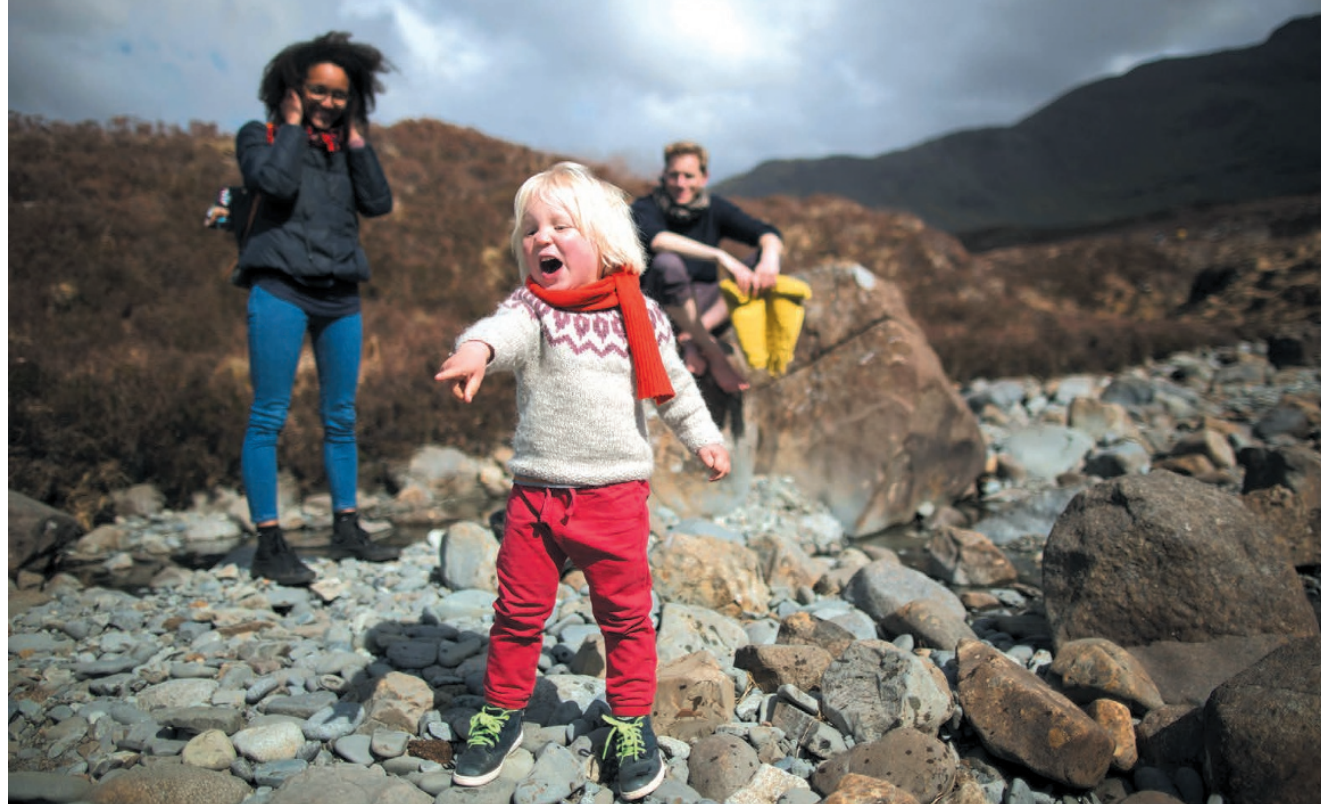

Pointing is an early milestone in children's communication.

LANGUAGE

\title{
Points, grunts and speaks
}

\section{Mark Pagel weighs up a study claiming that the origins of human language are rooted in gesture.}

\section{“A} nyone can speak Troll. All you have to do is point and grunt." Fred Weasley's assertion in J. K. Rowling's Harry Potter and the Goblet of Fire could describe the origins of human language. That is, if psychologist Michael Corballis is right in The Truth about Language. For years, Corballis has been one of the chief proponents of the idea that language has its origins in gestures. His latest book traces that argument from gesturing primates up through modern neuroscience.

Language is generally considered the jewel in the crown of human superiority over other animals. It seems to pop up almost out of nowhere in our evolutionary past, and no other animal's communication comes close to it. Through clicks, hoots, barks, chirrups and bleats, some animals can inform each other of how they are feeling. And some, through gestures, can signal anger, impatience, dominance or submission, desire and attachment. But none can use these displays to convey a simple sentence such as "I kicked the ball".

Being unique and powerful, human language is a siren to Darwinians, teasing us to examine how it arose, and from what. Corballis assigns a central role to the brain's mirror neurons, which seem to echo action in observation (in monkeys, mirror neurons fire when they reach to grasp objects, and when they observe another animal doing the same). Corballis points out that there is an overlap between parts of the mirror-neuron system and two areas in the brain's left cerebral cortex that are associated in humans with

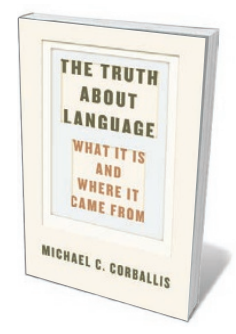

The Truth about Language: What It Is and Where It Came From MICHAEL

C. CORBALLIS

University of Chicago Press: 2017. the production and comprehension of language - Broca's and Wernicke's areas.

Mirror neurons have their detractors (see P. Smith Churchland Nature 511, 532-533; 2014), but for Corballis they raise the possibility that language "evolved within a system that, back in our monkey days, was specialised for grasping things". In humans, mirror neurons also seem to be involved with other actions. One is pointing. Conveniently for Corballis, this is one of the earliest milestones of communication in children ('look at that', 'please get that for me'), emerging around the first year, and signifying the beginning of shared attention. It is also disrupted in social disorders, such as autism. And the fine control of facial muscles required for speaking seems to share cortical circuitry with regions that control gestures.

Still, what is wrong with the view that our language grew from our hominid ancestors' capacity for vocalization? This was Charles Darwin's favourite explanation, put forth in The Descent of Man (1871). He thought that the capacity for complex vocal learning had deep evolutionary roots, extending at least as far back as our common ancestor with birds. Corballis counters that primate vocalizations, 
unlike gestures, seem barely under voluntary control. He contends that they emerge like nervous tics, more closely connected to expression of emotion than to a deliberate exchange of information. Vervet monkeys (Chlorocebus pygerythrus) famously have three different alarm calls, corresponding to predatory eagles, leopards and snakes. But an alarm call does not involve the give and take of a language, gestural or otherwise.

One suspects that many - such as the primatologist and ethologist Frans de Waal, author of Chimpanzee Politics (Johns Hopkins Univ. Press, 1983) — will find Corballis' assessment of primate vocalizations harsh. But primatologists such as Jane Goodall and David Premack share his views. Whatever the truth, Corballis writes with an academic's attention to detail in witty, self-deprecating prose. The combination of style and argument make The Truth about Language the best work yet on the gestural theory of language.

But what does it mean to say that language - the exchange of information between senders and receivers - arose from gestures? Gesturing may indicate a theory of mind, the ability to understand what others might know or be thinking; and surely that is a requisite for language. Humans take this understanding for granted, but it has proved difficult to show conclusively in other animals. Some dogs respond to pointing, but they have been selected to do so (and don't point things out to other dogs). Chimpanzees can point, but do it rarely: not what would be expected in routine social communication. Chimpanzees and other primates can follow others' gazes, but gazing is not an intentional act of communication. Indeed, following another's gaze is potentially an act of theft.

There the putative gestural trail goes cold in the long line of fossil hominins along the 6-million- to 7-million-year evolutionary path separating us from our common ancestor with chimpanzees. And yet this gap is where everything happened. Humans use language to promote learning, cooperation and the exchange of goods and services - a leap from occasional pointing. Our brand of sociality distinguishes our species as much as language does, and it is hard to imagine our social systems working without language.

One might equally argue that human language arose to exploit the psychological capabilities that make our advanced sociality possible. Corballis' position then has to be that gesturing provides the most likely evolutionary route to those capabilities. But this is a topic on which he says comparatively little. What we do know is that without those capabilities, we might still be pointing and grunting like the Trolls.

Mark Pagel is in the School of Biological Sciences at the University of Reading, UK. e-mail:m.pagel@reading.ac.uk

\section{Books in brief}

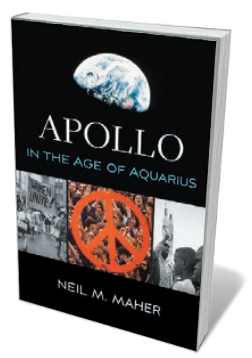

\section{Apollo in the Age of Aquarius}

Neil M. Maher HARVARD UNIVERSITY PRESS (2017)

On 20 July 1969, men landed on the Moon; back on Earth, the United States was gripped by sociopolitical convulsions. NASA and Woodstock may now seem polarized, but in this illuminating, original chronicle, historian Neil Maher traces multiple crosscurrents between them. The impact of the 'Blue Marble' image of Earth on environmental policy is famous (see go.nature.com/2ne4zai). Less so is how the costs of the Apollo programme enraged inner-city activists — and how NASA duly deployed a crack team of aeronautics experts to solve practical housing issues for poor African Americans.

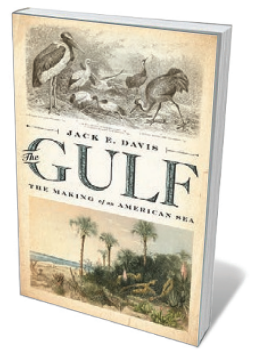

The Gulf: The Making of An American Sea

Jack E. Davis LIVERIGHT (2017)

In this assured ecological and human history, Jack Davis aims to rescue the Gulf of Mexico from its "hijacking" by the 2010

Deepwater Horizon oil spill. He argues that this 'sea' - with five US and six Mexican states lining its perimeter — has been more shaper than shaped, its unique biodiversity and geology inspiring currents of human development. It emerges as a beguiling entity, birthplace of the Gulf Stream, crucible of indigenous cultures, fishing mecca. Now, Davis avers, despite harbouring a mammoth hypoxic zone and ongoing petroleum extraction, the Gulf is in partial recovery.

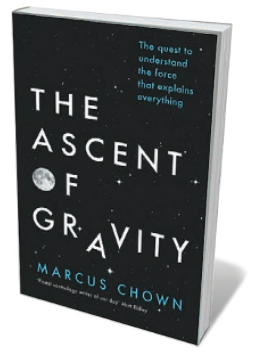

\section{The Ascent of Gravity}

Marcus Chown WIEDENFELD \& NICOLSON (2017)

"Everyone thinks it sucks but in most of the Universe it blows." That aphoristic introduction hints at the genial wit and scientific flair that await in Marcus Chown's primer on gravity, which traces the historic arc of our understanding of the force. He shows how Isaac Newton's 1687 Principia - which distilled fundamental laws from the complexity of the cosmos - helps to explain phenomena such as tides. He analyses Albert Einstein's reformulation of gravity as warped space-time. And he gazes into the weird realm of quantum theory - and the "undiscovered country" of the next big questions.

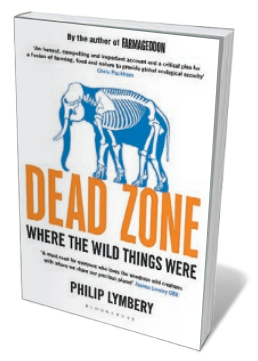

Dead Zone: Where the Wild Things Were

Philip Lymbery BLOOMSBURY (2017)

Half the planet's usable land is given over to agriculture, from crops to the 70 billion farm animals reared each year; meanwhile, an estimated half of all food is wasted. The impact on wildlife can be severe. In this measured analysis, Philip Lymbery — chief executive of the UK-based charity Compassion in World Farming - visits the front lines of industrial farming to gauge degrees of damage. Travelling from Sumatra's oil-palm monoculture, currently eating into elephant territory, to the encroachment of Brazilian soya fields on jaguar habitat, he argues that a transition to sustainable farming is overdue.

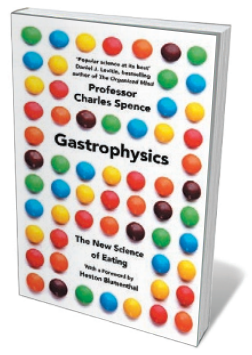

Gastrophysics: The New Science of Eating

Charles Spence VIKING (2017)

Cognitive psychologist Charles Spence opens his study on the science of gustation with an anecdote about being spoon-fed lime gelée at an acclaimed UK restaurant. As an illustration of how taste resides in the brain, it's faintly outré; but overall, there's much to savour in this detailed research round-up. Looking at sight, sound, touch and eating 'experience', Spence dishes up gems such as 'digital' chocolates, the link between tomato juice and aircraft noise, and the hyper-noisy packaging of Frito-Lay SunChips. Barbara Kiser 